\title{
THE SEMIMARTINGALE STRUCTURE OF REFLECTING BROWNIAN MOTION
}

\author{
RICHARD F. BASS AND PEI HSU
}

(Communicated by George C. Papanicolaou)

\begin{abstract}
We prove that reflecting Brownian motion on a bounded Lipschitz domain is a semimartingale. We also extend the well-known Skorokhod equation to this case.
\end{abstract}

In this note we study the semimartingale property and the Skorokhod equation of reflecting Brownian motion on a bounded Euclidean domain. A $R^{d}$. valued continuous stochastic process $X=\left\{X_{t} ; t \geq 0\right\}$ is said to be a semimartingale if it can be decomposed into the form

$$
X_{t}=X_{0}+M_{t}+\frac{1}{2} N_{t},
$$

where $M$ is a continuous martingale with zero initial value, and $N$ (ignoring the factor $1 / 2$ ) is a process of bounded variation. Let $|N|$ be its total variation process, i.e.,

$$
|N|_{t}=\sup \sum_{i=1}^{n-1}\left|N_{t_{i}}-N_{t_{i-1}}\right| .
$$

Here the supremum is taken over all finite partitions $0=t_{0}<t_{1}<\cdots<t_{n}=t$, and $|\cdot|$ denotes the Euclidean distance. We have the following expression

$$
N_{t}=\int_{0}^{t} \nu_{s} d|N|_{s}
$$

where $\nu$ is a process with length one, i.e., with probability one, $|\nu|_{s}=1$ for $|N|$-almost all $s$.

The original Skorokhod equation refers to one-dimensional reflecting Brownian motion $X=|B|$ (B is a standard one-dimensional Brownian motion). It states that $X$ is a semimartingale and $X_{t}=X_{0}+W_{t}+\frac{1}{2} L_{t}$, where $W$ is a standard Brownian motion and $L$ is the local time of $X$ at $x=0$.

Received by the editors July $17,1989$.

1980 Mathematics Subject Classification (1985 Revision). Primary 60J65; Secondary 60J35, $60 \mathrm{~J} 50$.

Key words and phrases. reflecting Brownian motion, Lipschitz domain, Dirichlet form, Skorokhod equation.

The first author was partially supported by NSF Grant DMS 88-22053; the second author was partially supported by NSF Grant DMS 86-01977. 
Suppose that $D$ is a bounded smooth domain in $R^{d}$. Let $\nu$ be the inward unit normal vector field on the boundary $\partial D$. Suppose that $X$ is a reflecting Brownian motion on $D$. The multidimensional Skorokhod equation takes the form

$$
X_{t}=X_{0}+W_{t}+\frac{1}{2} \int_{0}^{t} \nu\left(X_{s}\right) d L_{s},
$$

where $W$ is a standard $d$-dimensional Brownian motion and $L$ is the boundary local time of $X$, i.e., the continuous additive functional of $X$ associated with the surface measure of $D$. This form of the Skorokhod equation was first proved for convex domains in [T], then for $C^{1}$ domains by [LS] (see also [H]). In both cases, the stochastic Skorokhod equation is obtained by first solving a deterministic Skorokhod equation. As a matter of fact, (1) can be regarded as a stochastic differential equation with reflecting boundary conditions in two unknown processes $X$ and $L$. The existence and uniqueness of the solution of the deterministic Skorokhod equation imply the existence and pathwise uniqueness of the solution of the stochastic Skorokhod equation.

A natural question at this point is how smooth the domain $D$ has to be to insure that reflecting Brownian motion is a semimartingale. In this paper we will discuss bounded Lipschitz domains in any dimension. Our main result is that for these domains, reflecting Brownian motion is a semimartingale and the Skorokhod equation holds.

Let $D$ be a bounded Lipschitz domain. First we must make sure that reflecting Brownian motion can be defined as a continuous $\bar{D}$-valued process. This fact follows from our previous work $[\mathrm{BH}]$. For a discussion of reflecting Brownian motion on arbitrary domains, see [F1]. Further information on reflecting Brownian motion on Lipschitz and Hölder domains can be found in [BH].

Theorem 1. Suppose that $D$ is a bounded Lipschitz domain. Then reflecting Brownian motion $X$ is a continuous $\bar{D}$-valued semimartingale, and the Skorokhod equation

$$
X_{t}=X_{0}+W_{t}+\frac{1}{2} \int_{0}^{t} \nu\left(X_{s}\right) d L_{s},
$$

holds, where $W$ is a standard d-dimensional Brownian motion, $L$ is the boundary local time (continuous additive functional) associated with the surface measure $\sigma$ on $\partial D$, and $\nu$ is the inward unit normal vector field on the boundary.

The inward pointing normal vector is only defined a.e. (with respect to surface measure). However, the continuous additive functional $L$ is associated with $\sigma$ and so does not charge the null set. Hence the integral in the statement of Theorem 1 is unambiguously defined.

We will give a proof of Theorem 1 based on our previous work on reflecting Brownian motion on Lipschitz domains. For general domains, the reflecting Brownian motion may not be a continuous process on the Euclidean closure of $D$. It is a continuous process on a special compactification of $D$, the so-called 
Kuramochi compactification. In $[\mathrm{BH}]$, we have shown that if $D$ is a bounded Lipschitz domain, then the Kuramochi compactification of $D$ is the same as the Euclidean compactification. Thus, for such domains, the reflecting Brownian motion does live on the set $\bar{D}$. To show that it is actually a semimartingale, we use the theory of Dirichlet forms [F2].

Proof of Theorem 1. The Dirichlet form for reflecting Brownian motion is

$$
\mathscr{E}(u, v)=\frac{1}{2} \int_{D} \nabla u(x) \cdot \nabla v(x) m(d x), \quad D(\mathscr{E})=H^{1}(D) .
$$

( $m$ is the Lebesgue measure on $D$ ). In [BH] we proved that for $D$ bounded and Lipschitz, this Dirichlet form is regular on $\bar{D}$, which means that the set $H^{1}(D) \cap C(\bar{D})$ is dense in both $H^{1}(D)$ and $C(\bar{D})$, each functional space being equipped with its usual norm. We can now make use of the theory of regular Dirichlet forms developed in [F2], especially Chapter 5.

Suppose that $f \in H^{1}(D) \cap C(\bar{D})$. According to Theorem 5.2.2 of [F2], the continuous additive functional $f\left(X_{t}\right)-f\left(X_{0}\right)$ can be decomposed as follows:

$$
f\left(X_{t}\right)-f\left(X_{0}\right)=M_{t}^{f}+N_{t}^{f},
$$

where $M^{f}$ is a martingale additive functional of finite energy and $N^{f}$ is a continuous additive functional of zero energy. Since $X$ has continuous sample paths and $f$ is assumed to be continuous on $\bar{D}, M^{f}$ is a continuous martingale whose quadratic variation process is

$$
\left\langle M^{f}, M^{f}\right\rangle_{t}=\int_{0}^{t}|\nabla f|^{2}\left(X_{x}\right) d s .
$$

(See Example 5.2.1 in [F2].) If we further assume that $f \in C^{2}(\bar{D})$, then by Theorem 5.3.2 of [F2], $N^{f}$ is of bounded variation and its associated measure $\mu^{f}$ is uniquely characterized by the relation

$$
\frac{1}{2} \int_{D} \nabla f(x) \cdot \nabla v(x) m(d x)=\int_{\bar{D}} \tilde{v}(x) \mu^{f}(d x), \quad \forall v \in H^{1}(D) .
$$

( $\tilde{v}$ is a quasi-continuous modification of $v$.) Since $D$ is Lipschitz, we can use Green's identity in the above equation. This allows us to identify the associated measure of $N^{f}$, i.e.,

$$
\mu^{f}(d x)=-\frac{1}{2} \Delta f(x) m(d x)+\frac{1}{2} \frac{\partial f}{\partial \nu}(x) \sigma(d x),
$$

where $\sigma$ is the surface measure of the boundary $\partial D$.

Now apply the above discussion to the coordinate functions $f_{i}(x)=x^{i}$. We have

$$
X_{t}=X_{0}+M_{t}+\frac{1}{2} N_{t}
$$

where $M\left(M^{f_{1}}, \ldots, M^{f_{d}}\right)$, and $N=\left(N^{f_{1}}, \ldots, N^{f_{d}}\right)$. It remains to show that $M$ is a standard $d$-dimensional Brownian motion and $N_{t}=\int_{0}^{t} \nu\left(X_{s}\right) d L_{s}$. 
To see that $M$ is a Brownian motion, we use Lévy's criterion. Namely, we need to verify that

$$
\left\langle M^{f_{i}}, M^{f_{j}}\right\rangle=\delta_{i j} t, \quad i, j=1, \ldots, d .
$$

This follows immediately from (3). Therefore $M$ is a Brownian motion.

Let $\nu(x)=\left(\nu^{1}(x), \ldots, \nu^{d}(x)\right)$ be the components of the normal vector $\nu$. From (4), the measure associated with the continuous additive functional $N^{f_{i}}$ is $\nu^{i}(x) \sigma(d x)$. Let

$$
L_{t}=\sum_{i=1}^{d} \int_{0}^{t} \nu^{i}(x) d N_{s}^{f_{i}}
$$

It follows that the measure associated with $L$ is $\sum_{i=1}^{d} \nu^{i}(x)^{2} \sigma(d x)=\sigma(d x)$. This shows that $L$ is just the boundary local time with respect to the surface measure. Since the measure for $N^{f_{i}}$ is $\nu^{i}(x) \sigma(d x)$, we have

$$
N_{t}^{f_{i}}=\int_{0}^{t} \nu^{i}\left(X_{s}\right) d L_{s}, \quad i=1, \ldots, d .
$$

Hence we obtain

$$
N_{t}=\int_{0}^{t} \nu\left(X_{s}\right) d L_{s},
$$

and the proof of the Skorokhod equation is complete.

Remark. The tightness estimates of [BH, §2] allow us to construct reflecting Brownian motions on $\bar{D}$ when $D$ is a Hölder domain in $R^{d}, d \geq 3$. Unless the Kuramochi compactification for such a domain $D$ is equal to the Euclidean compactification, however, there will be more than one reflecting Brownian motion on $\bar{D}$, and the question of semimartingale representations loses some of its interest.

\section{REFERENCES}

[BH] R. F. Bass and P. Hsu, Some potential theory for reflecting Brownian motion in Hölder and Lipschitz domains (to appear in Ann. Probability).

[F1] M. Fukushima, A construction of reflecting barrier Brownian motions for bounded domains, Osaka J. Math. 4 (1967), 183-215.

[F2] __ Dirichlet forms and Markov processes, North-Holland, Amsterdam, 1980.

[H] P. Hsu, Reflecting Brownian motion, boundary local time, and the Neumann boundary value problem, Ph.D. dissertation, Stanford, 1984.

[LS] P. L. Lions and A. S. Sznitman, Stochastic differential equations with reflecting boundary conditions, Comm. Pure Appl. Math. 37 (1984), 511-537.

[T] H. Tanaka, Stochastic differential equations with reflecting boundary condition in convex region, Hiroshima Math. J. 9 (1967), 163-177.

Department of Mathematics, University of Washington, Seattle, Washington 98195

Department of Mathematics, Northwestern University, Evanston, Illinois 60208 